\section{Rendezvous biliary recanalization combining percutaneous and endoscopic techniques using a diathermic dilator for bile duct obstruction}
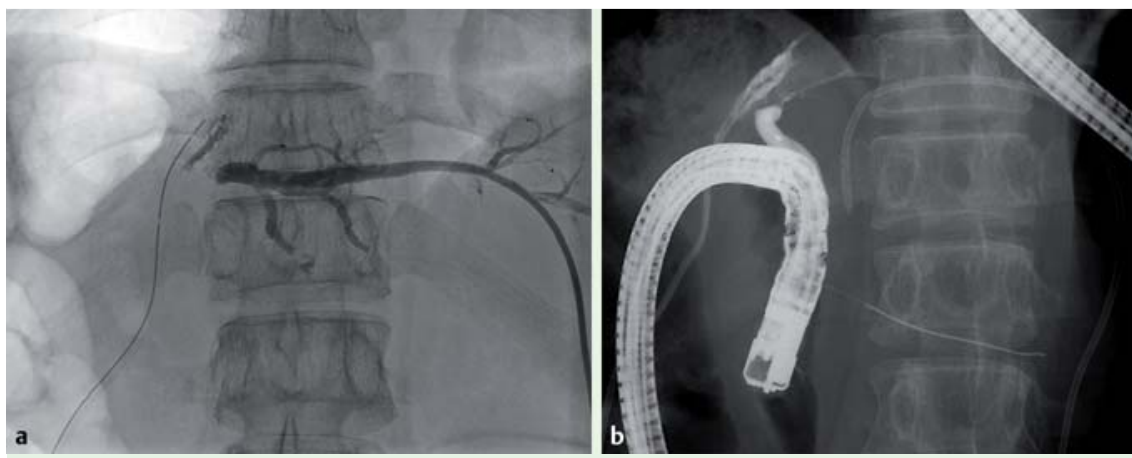

Fig. 1 Radiographic images showing a left hepatic bile duct obstruction after right hepatic trisectomy. a Percutaneous transhepatic cholangiography. b Endoscopic retrograde cholangiography .

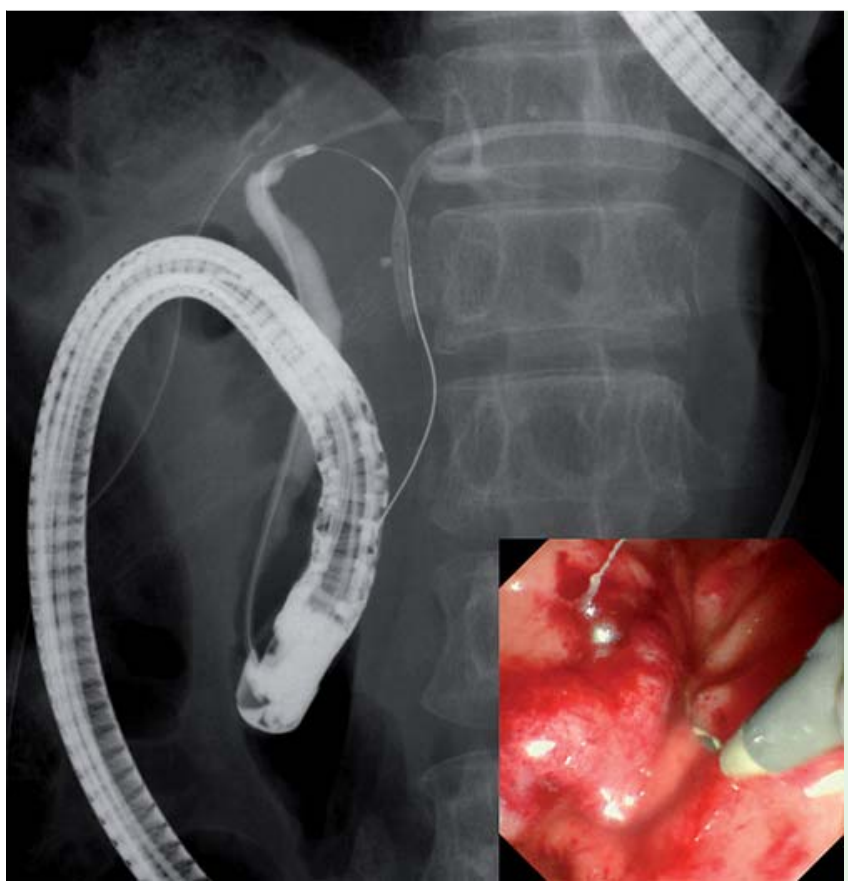

Fig. 3 Radiographic image showing the diathermic dilator being advanced over the guidewire to the level of the bile duct obstruction (inset: endoscopic view of the diathermic dilator being advanced over the guidewire into the bile duct).

Severe biliary injuries and strictures are intractable complications of hepatectomy. Endoscopic-radiologic biliary recanalization is a minimally invasive technique in patients undergoing hepatectomy. Here, we present a case of successful percutaneous transhepatic and endoscopic biliary rendezvous recanalization using a diathermic dilator for a benign severe postoperative biliary stricture.

A 64-year-old man with biloma and bile duct obstruction after right hepatic trisectomy for liver alveolar echinococcosis, was referred to our department. He had undergone percutaneous transhepatic biloma drainage and percutaneous trans- hepatic biliary drainage (PTBD) to the left dorsal branch 10 days previously. During PTBD, although the guidewire repeatedly entered the biloma, it could not be introduced into the left hepatic bile duct.

Percutaneous transhepatic cholangiography and endoscopic retrograde cholangiopancreatography (ERCP) revealed left hepatic bile duct obstruction ( $\bullet$ Fig. 1). A 0.025 -inch guidewire was successfully advanced across the stricture; however, a tapered ERCP catheter or dilator could not be advanced, and this made guidewire manipulation difficult ( Fig.2). A 6-Fr wire-guided diathermic dilator (CystoGastro-Set; Endo-Flex GmbH, Voerde,

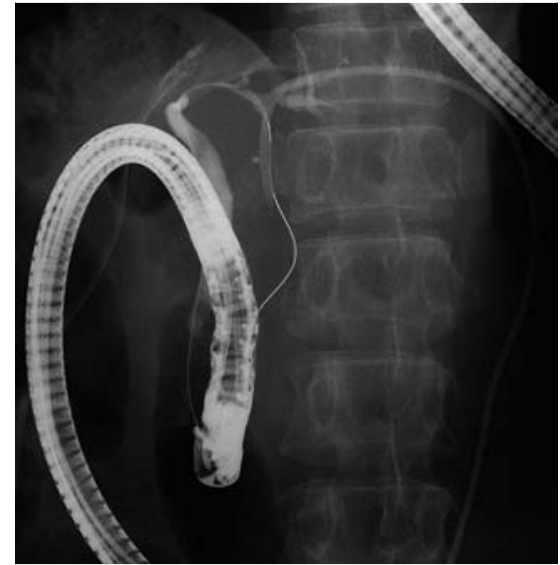

Fig.2 Radiographic image showing the hydrophilic 0.025 -inch guidewire that was successfully advanced across the left hepatic bile duct obstruction.

Germany) with a blended cut mode ( $\bullet$ Fig. 3; Video 1) was used, and the stricture was successfully dilated ( Fig. 4; - Video 1). Subsequently, the guidewire was grasped using the Amplatz GooseNeck Snare (10-mm; Covidien Japan, Tokyo, Japan) inserted via the PTBD route and pulled through ( $\bullet$ Fig. 5; Video 1). Communication between the left hepatic duct and common bile duct was established, and a new PTBD catheter was inserted over the guidewire without any complications ( Fig.6).

Endoscopic-radiologic rendezvous techniques for postoperative bile duct injury have been reported [1]. We recently reported the usefulness of a diathermic dilator for severe biliary strictures [2-5]. As described above, a diathermic dilator is useful for severe postoperative biliary strictures that cannot be dilated with various conventional techniques and for facilitating a rendezvous procedure.

\section{Endoscopy_UCTN_Code_TTT_1AR_2AG}

\section{Video 1}

Rendezvous biliary recanalization with combination of percutaneous and endoscopic techniques using a diathermic dilator for the left hepatic bile duct obstruction following right hepatic trisectomy. 

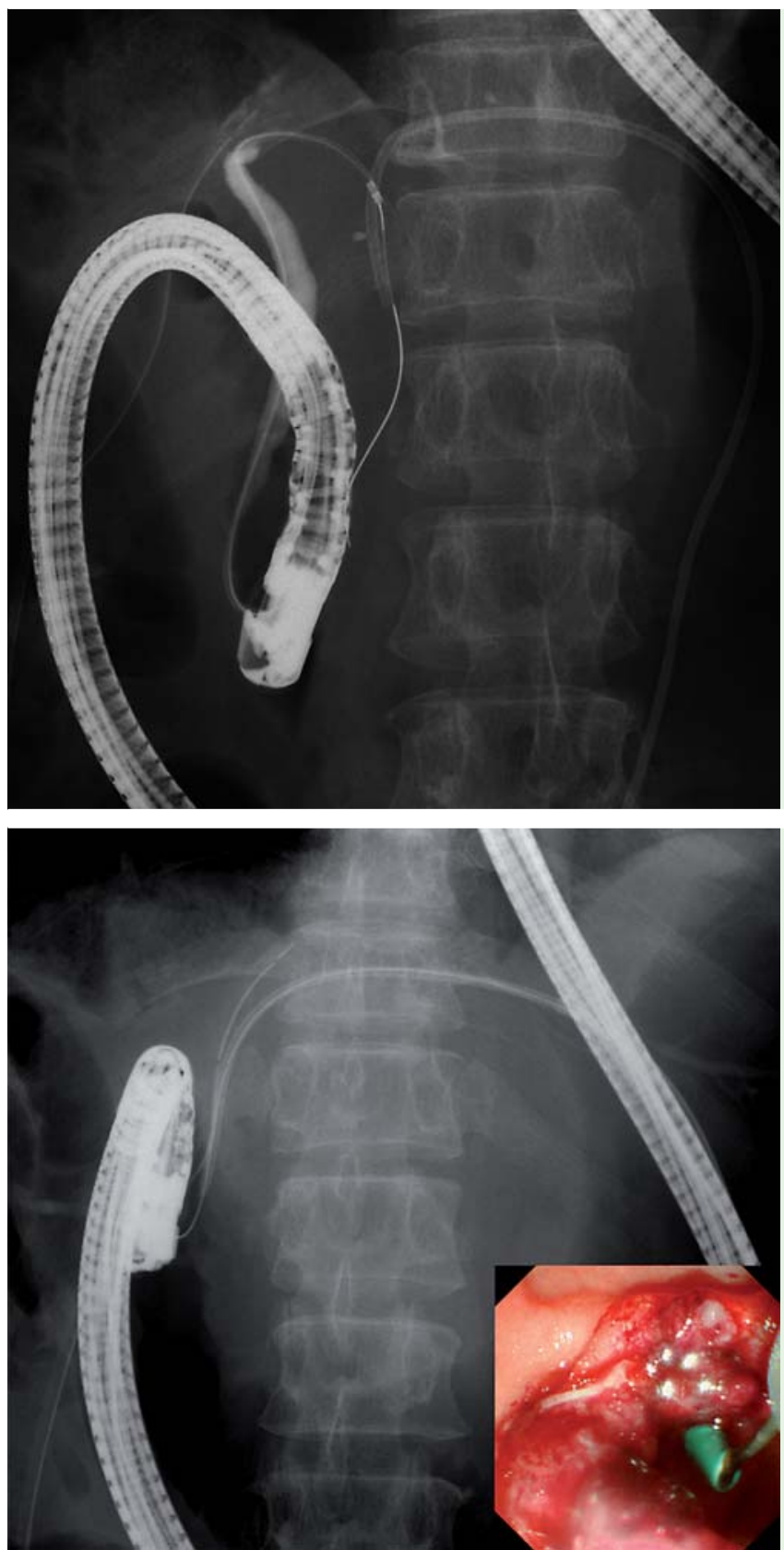

Competing interests: None

\section{Hiroshi Kawakami' ${ }^{1}$, Daisuke Abo², Kazumichi Kawakubo', Masaki Kuwatani ${ }^{1}$, Yuki Yoshino², Yoshimasa Kubota', Yoko Abe ${ }^{1}$, Shuhei Kawahata', Kimitoshi Kubo', Yusuke Sakuhara ${ }^{2}$, Hiroki Shirato², Naoya Sakamoto ${ }^{1}$}

${ }^{1}$ Department of Gastroenterology and Hepatology, Hokkaido University Graduate School of Medicine, Sapporo, Japan

2 Department of Radiation Medicine, Hokkaido University Graduate School of Medicine, Sapporo, Japan
Fig.4 Radiographic image showing the diathermic dilator being advanced across the biliary obstruction.

Fig. 6 Radiographic image showing a percutaneous transhepatic biliary drainage catheter being advanced across the biliary obstruction (inset: endoscopic view of a percutaneous transhepatic biliary drainage catheter being advanced over the guidewire through the ampulla of Vater to the duodenum).

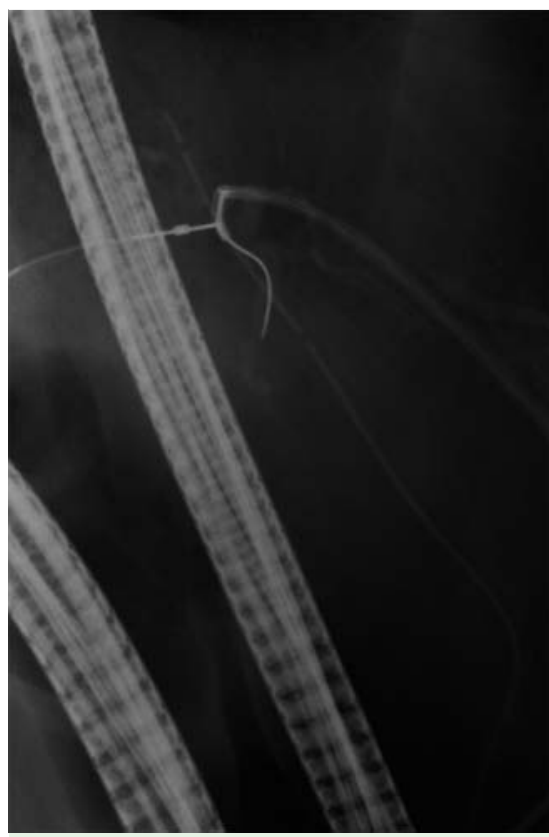

Fig. 5 Radiographic image confirming the communication between the left hepatic duct and common bile duct following the endoscopic and percutaneous rendezvous technique.

4 Kawakami H, Kuwatani M, Sakamoto $N$. Endoscopic ultrasound-guided antegrade diathermic dilation followed by self-expandable metallic stent placement for anastomotic stricture after hepaticojejunostomy (with video). Dig Endosc 2014; 26: $121-122$

5 Kawakami H, Kuwatani M, Kawakubo Ket al. Endoscopic ultrasound-guided antegrade diathermic dilation followed by self-expandable metallic stent placement for malignant distal biliary stricture. Endoscopy 2014. In press

\section{Bibliography}

DOI http://dx.doi.org/

10.1055/s-0034-1377553

Endoscopy 2014; 46: E460-E461

(C) Georg Thieme Verlag KG

Stuttgart · New York

ISSN 0013-726X plete transection of the main bile duct: minimally invasive treatment with an endoscopic-radiologic rendezvous. Gastrointest Endosc 2011; 74: 1393-1398

2 Kawakami H, Kuwatani M, Eto K et al. Resolution of a refractory severe biliary stricture using a diathermic sheath. Endoscopy 2012; 44 (Suppl. 02): E119-120

3 Kawakami H, Kuwatani M, Kawakubo Ket al. Transpapillary dilation of refractory severe biliary stricture or main pancreatic duct by using a wire-guided diathermic dilator (with video). Gastrointest Endosc 2014; 79: $338-343$

\section{Corresponding author}

\section{Hiroshi Kawakami, MD, PhD}

Department of Gastroenterology and Hepatology Hokkaido University Graduate School of Medicine Kita 15, Nishi 7

Kita-ku

Sapporo 060-8638

Japan

Fax: +81-11-7067867

hiropon@med.hokudai.ac.jp 